\title{
SMOOTH SETS FOR A BOREL EQUIVALENCE RELATION
}

\author{
CARLOS E. UZCÁTEGUI A.
}

\begin{abstract}
We study some properties of smooth Borel sets with respect to a Borel equivalence relation, showing some analogies with the collection of countable sets from a descriptive set theoretic point of view. We found what can be seen as an analog of the hyperarithmetic points in the context of smooth sets. We generalize a theorem of Weiss from Z-actions to actions by arbitrary countable groups. We show that the $\sigma$-ideal of closed smooth sets is $\Pi_{1}^{1}$ non-Borel.
\end{abstract}

\section{INTRODUCTION}

The study of Borel equivalence relations have received recently considerable attention from the descriptive set theoretic standpoint (see [6], [11], [3] and the references therein). In this paper we will present some results about smooth sets, a notion of smallness naturally associated with an equivalence relation. Smooth sets are a generalization of wandering sets, which appear in ergodic theory in the study of the action of an homeomorphism over a Polish space ([16]). These collections of negligible sets ("small" sets like measure zero sets or meager sets) form a $\sigma$-ideal and they occur quite naturally in many areas of mathematics. One such $\sigma$-ideal that has been studied quite well in descriptive set theory (and became a sort of a paradigm) is the $\sigma$-ideal of countable sets. Smooth sets have some properties similar to those of the collection of countable sets, in particular, several of its features can be deduced by analyzing the collection of compact smooth sets. The study of a $\sigma$-ideal $I$ by looking at the compact sets in $I$ has been the focus of much work since the discovery of the connection of some problems in harmonic analysis (about set of uniqueness) with the structure of $\sigma$-ideals of compact sets (see for instance [12] and [14]).

Let us recall the definition of smooth sets ([6]). Let $X$ be a Polish space (i.e., a complete separable metric space). An equivalence relation $E$ over $X$ is called Borel if $E$ is a Borel set as a subset of $X \times X$ and it is said to be smooth if it admits a countable Borel separating family, i.e., a collection $\left(A_{n}\right)$

Received by the editors September 6, 1993 and, in revised form, August 4, 1994.

1991 Mathematics Subject Classification. Primary 03E15, 04A15, 54H20; Secondary 28A05, 28D99, 54H05.

Key words and phrases. Borel equivalence relations, negligible sets, $\sigma$-ideals of compact sets, group actions, wandering sets. 
of $E$-invariant Borel subsets of $X$ such that for all $x, y \in X$

$$
x E y \text { if and only if } \forall n\left(x \in A_{n} \leftrightarrow y \in A_{n}\right) .
$$

A Borel equivalence relation is smooth if it admits definable invariants, that is, one can assign in a Borel way to each equivalence class an invariant (an element of some Polish space, [6]). The best case would be when the invariant is an element of the equivalence class itself, i.e., when there is a Borel transversal (but this is not always the case). Given an arbitrary Borel equivalence relation $E$ on $X$, a set $A \subseteq X$ is called $E$-smooth if there is a Borel set $B \supseteq A$ such that the restriction of $E$ to $B$ is a smooth equivalence relation. The collection of $E$-smooth sets forms a $\sigma$-ideal and thus they will be considered "small" sets. A fundamental result in Borel equivalence relations is the Glimm-Effros type dichotomy theorem proved by Harrington, Kechris and Louveau ([6]), which characterizes the smooth Borel equivalence relations and thus the Borel smooth sets.

This paper is organized as follows: In $\S 2$ we show an extension to analytic sets of the Glimm-Effros type dichotomy theorem (Theorem 2.3). In order to follow the proofs of some of the results on this section, the reader must be familiar with the Harrington-Kechris-Louveau paper (they heavily use the tools of effective descriptive set theory, especially the Gandy-Harrington topology). Theorem 2.3 can be considered as an analog of the perfect set theorem in the context of smoothness. We present what can be seen as an analog of the hyperarithmetic reals (see Theorem 2.7 (iii) and Theorem 4.7). Theorem 2.3 will also provide the basic representation of $\Sigma_{1}^{1}$ smooth sets as the common null sets for the family of $E$-ergodic non-atomic measures. In particular, it says that smoothness for $\Sigma_{1}^{1}$ sets is a notion concentrated on closed sets, i.e., a $\Sigma_{1}^{1}$ set $A$ is smooth if and only if every closed subset of $A$ is smooth. In general, we called a set sparse if every closed subset of it is smooth. Every smooth set is sparse. However, a result of Kechris and Becker shows that not every co-analytic sparse set is smooth. We will present the proof of this result in $\S 3$.

In $\S 4$ we will look at the particular case of a countable equivalence relation (i.e., one all of whose equivalence classes are countable). We generalize a theorem of Weiss ([16]) (which characterizes smooth equivalence relations induced by the action of an homeomorphism) to the case of a countable Borel equivalence relation. We show that in general smooth sets are not necessarily of first category.

Since smoothness for analytic sets is concentrated on closed sets we will look in $\S 5$ at the $\sigma$-ideal of closed smooth sets. Following ideas from [14] and [18] we will show that it is a strongly calibrated, locally non-Borel, $\Pi_{1}^{1} \sigma$-ideal.

Most of the results presented in this paper are part of my Ph.D. thesis. I would like to thank my adviser Dr. Alexander Kechris for his guidance and patience. I would also like to thank the anonymous referee for his (her) helpful comments.

\section{SмоOTH SETS}

First we will define some basic concepts and state some basic facts. Our notation is standard as in [15] and all descriptive set theoretic concepts not defined in this paper can be found in Moschovakis' book. Let $X$ be a Polish 
space (i.e., a complete separable metric space), since we work with effective methods we ask that $X$ is recursively presented ([15]). $\Sigma_{1}^{1}$ denotes the analytic sets, $\Pi_{1}^{1}$ the co-analytic sets and $\Delta_{1}^{1}$ the Borel sets. The corresponding effective point-classes are denoted respectively by $\Sigma_{1}^{1}, \Pi_{1}^{1}$ and $\Delta_{1}^{1} . E$ will always denote a Borel equivalence relation on $X .[x]_{E}$ or sometimes $E_{x}$ will denote the $E$ equivalence class of $x .[A]_{E}$ is the saturation of $A$, i.e., $[A]_{E}=\{y \in X$ : $\exists x \in A(x E y)\}$. A set $A$ is called $E$-invariant (or just invariant, if there is no confusion about $E$ ) if $A=[A]_{E}$. The restriction of $E$ to a subset $A$ is denoted by $E\left\lceil A\right.$. Given a $\Delta_{1}^{1}$ equivalence relation $E$ (i.e., $E$ as a subset of $X \times X$ is a $\Delta_{1}^{1}$ set) and $A \subseteq B$, with $B$ a $\Pi_{1}^{1}$ invariant set and $A$ a $\Sigma_{1}^{1}$ set, then there is a $\Delta_{1}^{1}$ invariant set $C$ with $A \subseteq C \subseteq B$. In other words, the separation theorem holds in an invariant form for $\Delta_{1}^{1}$ equivalence relations (actually it holds for $\Sigma_{1}^{1}$ equivalence relations). A proof of this can be found in [6] (Lemma 5.1). We will use the following notation: Script capital letters will denote a countable family of subsets of $X$, i.e., $\mathscr{A}=\left(A_{n}\right)$, with $A_{n} \subseteq X$ for $n \in \mathbf{N}$. For each collection $\mathscr{A}$ we define the following equivalence relation:

$$
x E_{\mathscr{A}} y \text { if and only if } \forall n\left(x \in A_{n} \longleftrightarrow y \in A_{n}\right) .
$$

Definition 2.1. Let $\Gamma$ be a point-class.

(i) $E$ is $\Gamma$-separated if and only if there is a countable collection $\mathscr{A}=\left(A_{n}\right)$ with each $A_{n} \in \Gamma$, such that: $\forall x \forall y\left(x E y \longleftrightarrow x E_{\mathscr{A}} y\right)$, i.e., $E=E_{\mathscr{A}}$.

(ii) A subset $A$ of $X$ is $\Gamma$-separated, if and only if there is a collection $\mathscr{A}=$ $\left(A_{n}\right)$ of $E$-invariant sets, with each $A_{n} \in \Gamma$, and $\forall x \in A, \forall y \in A(x E y \longleftrightarrow$ $x E_{\mathscr{A}} y$ ). In this case we say that $\mathscr{A}$ separates $A$.

(iii) $A$ is called strongly $\Gamma$-separated if $\forall x \in A \forall y\left(x E y \longleftrightarrow x E_{\mathscr{A}} y\right)$; and we say that $\mathscr{A}$ strongly separates $A$.

Remark. (1) Notice that in (i), each $A_{n}$ has to be $E$-invariant (because if $x \in$ $A_{n}$ and $y E x$, then $x E_{\mathscr{A}} y$. Hence $\left.y \in A_{n}\right)$.

(2) Denote by $[x]_{\mathscr{A}}$ the $E_{\mathscr{A}}$-equivalence class of $x$. Then $\mathscr{A}$ separates $A$ if and only if for all $x \in A,[x]_{E} \cap A=[x]_{\mathscr{A}} \cap A$; and $\mathscr{A}$ strongly separates $A$ if and only if for all $x \in A,[x]_{E}=[x]_{\mathscr{A}}$. We will see later that Borel separation and strong Borel separation are equivalent notions for analytic sets but are not equivalent for co-analytic sets.

(3) If $\mathscr{A}=\left(A_{n}\right)$ and each $A_{n}$ is invariant then $E \subseteq E_{\mathscr{A}}$, thus only one direction in (ii) is not trivial.

(4) Let $A \in \Gamma$ be an invariant set and $\Gamma$ closed under intersections, then it is clear that $A$ is $\Gamma$-separated if and only if $E\lceil A$ is $\Gamma$-separated.

As we said in the introduction, a Borel equivalence relation is called smooth if it is Borel separated. A finite, positive Borel measure $\mu$ on $X$ is called $E$ ergodic (or just ergodic) if for every $\mu$-measurable invariant set $A, \mu(A)=0$ or $\mu(X-A)=0$. It is called $E$-non-atomic (or just non-atomic), if for every $x \in$ $X \mu\left([x]_{E}\right)=0$. For the restriction of an equivalence relation to a set we define the corresponding notions as follows: A measure $\mu$ is called $E\lceil A$-ergodic if $\mu(X-A)=0$ and for every $B \subseteq A$ which is $E\lceil A$-invariant and $\mu$-measurable, we have $\mu(B)=0$ or $\mu(X-B)=0$. Notice that in this case $\mu(X-B)=0$ if and only if $\mu(A-B)=0$. A basic fact about $E$-ergodic non-atomic measure is that if $\mu$ is such a measure, then there is no $\mu$-measurable separating family 
for $E$. A typical example of an equivalence relation with a non-atomic ergodic measure is $E_{0}$, which is defined on $2^{\omega}$ by

$$
x E_{0} y \text { if and only if } \exists m \forall n>m x(n)=y(n) .
$$

The usual product measure on $2^{\omega}$ is non-atomic and $E_{0}$-ergodic (the so-called 0-1 law).

One way of defining ergodic measures is through an embedding. Let $E$ and $E^{\prime}$ be two equivalence relations on $X$ and $Y$ respectively. An embedding from $E$ into $E^{\prime}$ is a 1-1 map $f: X \rightarrow Y$ such that for all $x, y \in X, x E y \longleftrightarrow$ $f(x) E^{\prime} f(y)$. For Borel equivalence relations we define $E \sqsubseteq E^{\prime}$ if there is a Borel embedding of $E$ into $E^{\prime}$.

The fundamental result about the notion of smoothness is the following

Theorem 2.2 (Harrington, Kechris, Louveau [6]). Let $X$ be a recursively presented perfect Polish space, $E$ a $\Delta_{1}^{1}$ equivalence relation on $X$. Then exactly one of the following holds:

(i) $E$ has a $\Delta_{1}^{1}$ separating family $\mathscr{A}=\left(A_{n}\right)$, such that the set $(x, n) \in A \Leftrightarrow$ $x \in A_{n}$ is $\Delta_{1}^{1}$ in $X \times \omega$.

(ii) $E_{0} \sqsubseteq E$ (via a continuous embedding).

The next theorem says, among other things, that for $\Sigma_{1}^{1}$ sets all natural variations of countable separation are equivalent.

Theorem 2.3. Let $X$ be a recursively presented Polish space, $E$ a $\Delta_{1}^{1}$ equivalence relation on $X$, and $A$ a $\Sigma_{1}^{1}$ subset of $X$. The following are equivalent:

(i) There is a $\Delta_{1}^{1}$ invariant set $B$ such that $A \subseteq B$ and $B$ is strongly $\Delta_{1}^{1}$ separated. Moreover, the separating family $\mathscr{A}=\left(\bar{A}_{n}\right)$ for $B$ is uniformly $\Delta_{1}^{1}$, i.e., the set $(x, n) \in A \Leftrightarrow x \in A_{n}$ is $\Delta_{1}^{1}$ in $X \times \omega$.

(ii) $A$ is strongly $\Delta_{1}^{1}$-separated.

(iii) $[A]_{E}$ is $\Sigma_{1}^{1}$-separated.

(iv) $A$ is $\Sigma_{1}^{1}$-separated.

(v) $E\left\lceil A\right.$ is $\Sigma_{1}^{1}$-separated.

(vi) $A$ is universally measurable separated.

(vii) $E[A$ is universally measurable separated.

(viii) For every $E$-ergodic non-atomic measure $\mu, \mu(A)=0$.

(ix) For every $E\lceil A$-ergodic, non-atomic measure $\mu, \mu(A)=0$.

(x) $E_{0} Z E\lceil A$.

Similarly, the same equivalences hold by relativization for a $\Sigma_{1}^{1}$ set $A$ and a $\Delta_{1}^{1}$ equivalence relation.

All the equivalences are more or less straightforward, except for $(\mathbf{x}) \Rightarrow$ (i) which uses two results proved in [6]. As we said in the introduction we assume that the reader is familiar with the Harrington-Kechris-Louveau paper [6]. We will need the following lemmas.

Lemma 2.4. Let $\tau$ be the Gandy-Harrington topology on $X$ and $\bar{E}$ the $\tau \times \tau$ closure of $E$. Let $A$ be a $\Sigma_{1}^{1}$ subset of $X$. If $\left\{x: E_{x} \neq(\bar{E})_{x}\right\} \cap A \neq \varnothing$ then $E_{0} \sqsubseteq E\lceil A$, via a continuous embedding. 
Proof. By Lemma 5.3 of [6] $\left\{x: E_{x} \neq(\bar{E})_{x}\right\} \cap A \neq \varnothing$, then $E$ is meager in $(A \times A) \cap \bar{E}$. Hence the construction of the embedding from $E_{0}$ into $E\lceil A$ can be carried out in $A$.

Lemma 2.5. Let $D=\left\{x: E_{x}=(\bar{E})_{x}\right\}, D$ is a $\Pi_{1}^{1}$ strongly $\Delta_{1}^{1}$-separated invariant set. Moreover, the separating family for $D$ is $\left\{A \subseteq X: A\right.$ is a $\Delta_{1}^{1}$ invariant set\}.

Proof. First, $\bar{E}$ is a $\Sigma_{1}^{1}$ equivalence relation (Lemma 5.2 of [6]). We have that $x \in D$ if and only if $\forall y(x \bar{E} y \rightarrow x E y)$. Thus $D$ is $\Pi_{1}^{1}$. Also, as $E \subseteq \bar{E}$, then $D$ is $E$-invariant (actually $\bar{E}$-invariant). On the other hand, we know $\bar{E}=\sim \bigcup\left\{A \times \sim A: A\right.$ is $\Delta_{1}^{1}$ invariant set $\}$. So, if $\mathscr{A}=\left\{A: A\right.$ is a $\Delta_{1}^{1}$ invariant set $\}$, then $\bar{E}=E_{\mathscr{A}}$. And we get: $\forall x \in D\left(E_{x}=(\bar{E})_{x}=\left(E_{\mathscr{A}}\right)_{x}\right)$. Thus $\forall x \in D \forall y\left(x E_{\mathscr{A}} y \longleftrightarrow x E y\right)$, i.e., $D$ is strongly separated by $\mathscr{A}$.

Proof of Theorem 2.3. Now we finish the proof of $(\mathbf{x}) \Rightarrow$ (i). Suppose $(\mathbf{x})$ holds. Then by Lemma $2.4 A \subseteq D$. By separation (Lemma 5.1 [6]) there is a $\Delta_{1}^{1}$ invariant set $B$ with $A \subseteq B \subseteq D$. Hence, by Lemma $2.5 B$ is strongly $\Delta_{1}^{1}$ separated by $\mathscr{A}=\left\{A \subseteq X: A\right.$ is $\Delta_{1}^{1}$ invariant set $\} . \mathscr{A}$ is clearly a $\Pi_{1}^{1}$ collection, so by a separation argument (page 922, [6]) we can easily show that there is a $\Delta_{1}^{1}$ subsequence of $\mathscr{A}$ which also separates $B$, so (i) holds.

In view of this result we have

Definition 2.6. (i) Let $E$ be a Borel equivalence relation on $X$. A $\Sigma_{1}^{1}$ subset $A \subseteq X$ is called $E$-smooth if any of the equivalent conditions of Theorem 2.3 holds.

(ii) A set $A \subseteq X$ is called $E$-smooth if there is a Borel smooth set $B$ such that $A \subseteq B$.

It is clear that a subset of a smooth set is also smooth and a countable union of smooth sets is smooth, i.e., they form a $\sigma$-ideal. So, we regard smooth sets as small sets. Every countable set is smooth and $E$ is smooth iff $X$ is smooth. Other very simple smooth sets are the Borel transversals: A set $A$ is called an $E$-transversal (or just a transversal) if for all $x, y \in A$ with $x \neq y$ we have $x B x$. It is easy to see that every Borel (even analytic) transversal is $\boldsymbol{\Sigma}_{1}^{1}$ separated (in fact, let $T$ be an analytic transversal, $V_{n}$ be an open basis for the topology of $X$ and put $A_{n}=\left[T \cap V_{n}\right]_{E}$, then $\left(A_{n}\right)$ is a separating family for $T$ ). We say that a transversal $T$ is total if its saturation $[T]_{E}$ is the whole space $X$ (in this case $E$ is a smooth equivalence relation). The standard proof that there is a non-Lebesgue measurable set goes by showing that the following equivalence relation does not admit a (total) Lebesgue measurable transversal: $X$ is the unit interval and $x E y$ if $x-y$ is a rational number. In fact, this equivalence relation is not smooth.

There is a strong similarity between the collection of countable sets and the collection of $\Sigma_{1}^{1}$ smooth sets, which is summarized in the following:

Theorem 2.7. Let $E$ be a $\Delta_{1}^{1}$ equivalence relation on a recursively presented Polish space $X$.

(i) (Analog of the perfect set theorem for $\Sigma_{1}^{1}$ sets) Let $A \subseteq X$ be a $\Sigma_{1}^{1}$ set. Then either $A$ is smooth or $E_{0} \sqsubseteq E\lceil A$ (via a continuous embedding). Similarly 
the same result holds by relativization for a $\Sigma_{1}^{1}$ set $A$ and a $\Delta_{1}^{1}$ equivalence relation $E$.

(ii) The collection of $\Sigma_{1}^{1}$ smooth sets is $\Pi_{1}^{1}$ on the codes of $\Sigma_{1}^{1}$ sets.

(iii) (Analog of the hyperarithmetic reals) Let $\bar{E}$ be the $\tau \times \tau$-closure of $E$, where $\tau$ is the Gandy-Harrington topology on $X$. Put

$$
D=\left\{x: E_{x}=(\bar{E})_{x}\right\} .
$$

Then $D$ is a $\Pi_{1}^{1}$ set and for every $\Sigma_{1}^{1}$ set $A, A$ is smooth if and only if $A \subseteq D$. Proof. (i) It follows from Theorem 2.3.

(ii) Let $\mathscr{U}$ be a $\Sigma_{1}^{1}$ universal set. Then from Theorem 2.3 we have that

$$
\mathscr{U}_{\alpha} \text { is smooth iff } \exists \mathscr{A} \in \Delta_{1}^{1}(\alpha) \forall x, y \in \mathscr{U}_{\alpha}\left(x E y \longleftrightarrow x E_{\mathscr{A}} y\right) \text {. }
$$

It is easy to see that the relation above is a $\Pi_{1}^{1}$ relation in $\alpha$ by coding sequences of $\Delta_{1}^{1}(\alpha)$ invariants sets and using the theorem of restricted quantification (4D.3 in $[15])$.

(iii) It follows from Lemma 2.4 and Lemma 2.5.

Remark. (1) The set $D$ is the largest strongly $\Delta_{1}^{1}$-separated set, in fact: Let $\mathscr{A}=$ $\left\{A: A\right.$ is $\Delta_{1}^{1}$ invariant set $\}, B$ a strongly $\Delta_{1}^{1}$-separated set and $\mathscr{B}$ a family of $\Delta_{1}^{1}$-invariant sets that strongly separates $B$. Let $D_{\mathscr{B}}=\left\{x:[x]_{E}=[x]_{\mathscr{B}}\right\}$, i.e., $x \in D_{\mathscr{B}}$ if and only if for all $y\left(x E_{\mathscr{B}} y \longleftrightarrow x E y\right)$, and define analogously $D_{\mathscr{A}}$. We saw in 2.5 that $D=D_{\mathscr{A}}$. By definition of strong separation $B \subseteq D_{\mathscr{B}}$. But as $\mathscr{B} \subseteq \mathscr{A}$, then $E_{\mathscr{A}} \subseteq E_{\mathscr{B}}$ and thus $D_{\mathscr{B}} \subseteq D_{\mathscr{A}}$. Therefore $B \subseteq D_{\mathscr{A}}$.

(2) Recall that the collection of hyperarithmetic points, denoted by $\Delta_{1}^{1}(X)$, has the property that for every $\Sigma_{1}^{1}$ set $A \subseteq X, A$ is countable iff $A \subseteq \Delta_{1}^{1}(X)$ (see 4F.1 in [15]). This is the reason why $D$ is called an analog of the hyperarithmetic points. $\Delta_{1}^{1}(X)$ is a true $\Pi_{1}^{1}$ set (see $4 D .16$ in [15]) and is equal to $\bigcup\left\{A: A\right.$ is a countable $\Delta_{1}^{1}$ set $\}$. These analogies suggest the following questions:

(i) Is $D=\bigcup\left\{A: A\right.$ is $\Delta_{1}^{1}$ smooth set $\}$ ? Equivalently, is $D$ the union of $\Sigma_{1}^{1}$ sets?

(ii) Is $D$ a true $\Pi_{1}^{1}$ set ?

We will show in $\S 3$ that for a countable $\Delta_{1}^{1}$ equivalence relation the answer for (i) is yes (in fact, as a consequence of a theorem of Kechris, this is also true for a $\Delta_{1}^{1}$ equivalence relation generated by the action of a locally compact group of $\Delta_{1}^{1}$ automorphisms of $X$, see [17]). Regarding question (ii), $D$ (for $E_{0}$ ) has measure zero with respect to the standard product measure on $2^{\omega}$ (because this measure is $E_{0}$-ergodic). Also every $\Delta_{1}^{1}$ point $x \in 2^{\omega}$ belongs to $D$ (since $\{x\}$ is a $\Delta_{1}^{1}$ smooth set). Then by a basis theorem (Corollary 4.2 in [10]) $D$ cannot be $\Delta_{1}^{1}$, otherwise its complement would contain a $\Delta_{1}^{1}$ point. Hence in this case $D$ is a true $\Pi_{1}^{1}$ set and the analogy between $D$ and the hyperarithmetic points is quite clear.

\section{SPARSE SETS}

We have shown (Theorem 2.7) the similarities between analytic smooth sets and countable sets. In general, however, we cannot say the same for co-analytic sets, as we will see next. A set is called $E$-sparse (or just sparse) if every closed 
subset of it is $E$-smooth. Sparse sets are the analog of thin sets (i.e., sets without perfect subsets). From 2.3 we have that every smooth set is sparse and that $A$ is $E$-sparse if and only if $E_{0} \square E\lceil A$. Notice also that if $A$ is universally measurable (for instance co-analytic) then $A$ is $E$-sparse if and only if for every $E$-ergodic non-atomic measure $\mu$ in $X$ we have $\mu(A)=0$ (i.e, (viii) in Theorem 2.3 holds). However it is not necessarily true that $A$ is contained in a Borel smooth set (i.e., (i) in Theorem 2.3 does not hold).

The following result was first proved by $\mathrm{H}$. Becker [1] using $\Delta_{2}^{1}$-determinacy. We present a proof due to A. Kechris [13]. I would like to thank them for allowing the presentation of their result in this paper. Let $\Delta(X)$ be the identity relation on $X$.

Theorem 3.1 (Becker, Kechris). Consider the equivalence relation $E=\Delta\left(\omega^{\omega}\right) \times$ $E_{0}$ on $\omega^{\omega} \times 2^{\omega}$. There is a $\Pi_{1}^{1}$ subset of $\omega^{\omega} \times 2^{\omega}$ which is $E$-sparse but not E-smooth. In fact, there is a $\mathbf{\Pi}_{1}^{1}$ transversal which is Borel separated but not smooth.

Proof. Let $S$ be $\Sigma_{1}^{1}$ and $P$ be $\Pi_{1}^{1}$ subset of $\omega^{\omega} \times\left(\omega^{\omega} \times 2^{\omega}\right)$ universal for $\Sigma_{1}^{1}$ and $\Pi_{1}^{1}$ subsets of $\omega^{\omega} \times 2^{\omega}$, respectively. Let $C \subset \omega^{\omega}$ be a $\Pi_{1}^{1}$ set of codes for the Borel subsets of $\omega^{\omega} \times 2^{\omega}$, i.e., if $x \in C$ then $S_{x}=P_{x}\left(=D_{x}\right)$ and $\left\{D_{x}: x \in C\right\}=\Delta_{1}^{1}\left(\omega^{\omega} \times 2^{\omega}\right)$. Put

$$
x \in B \Longleftrightarrow x \in C \& D_{x} \text { is smooth. }
$$

By Theorem 2.7 (ii) we know that $B$ is $\Pi_{1}^{1}$. Put next

$$
(x, y) \in A^{\prime} \Longleftrightarrow x \in B \&(x, y) \notin D_{x} .
$$

For each $x \in B$ we have that $\left(A^{\prime}\right)_{x} \neq \varnothing$, since if $R$ is $\Delta\left(\omega^{\omega}\right) \times E_{0}$-smooth, then for every $x \in \omega^{\omega} \quad R_{x}$ is $E_{0}$-smooth. Let $A$ be a $\Pi_{1}^{1}$-uniformizing subset of $A^{\prime}$ ([15]). Clearly, $A$ is a partial transversal for $\Delta\left(\omega^{\omega}\right) \times 2^{\omega}$, i.e., if $a, b \in A$ and $a \neq b$ then $(a, b) \notin \Delta\left(\omega^{\omega}\right) \times E_{0}$, so $A$ is sparse. If, towards a contradiction, $A \subset D$ where $D$ is Borel smooth, let $x \in C$ such that $D_{x}=D$. Then clearly $x \in B$. Let $(x, y) \in A$, so $(x, y) \in D_{x}=D$, a contradiction.

Notice that $A$ is Borel separated, in fact let $V_{n}, W_{n}$ be open bases for $\omega^{\omega}$ and $2^{\omega}$ respectively. Let $A_{n, m}=V_{n} \times\left[W_{m}\right]_{E}$. Then it is easy to check that $\left(A_{n, m}\right)$ is a Borel separating family for $A$.

Remark. We will see in the next section that the set $A$ in the previous proof is not strongly Borel separated.

Theorem 2.7 (i) is a perfect set type theorem for analytic smooth sets. The previous result shows that such a theorem cannot be extended to co-analytic sets. This is an essential difference between sparse sets and thin sets (recall that a theorem of Solovay says that if there is an inaccessible cardinal then it is consistent that every $\boldsymbol{\Pi}_{1}^{1}$ thin set is countable). There is another structural property of the co-analytic thin sets that has been studied, namely the existence of the largest $\Pi_{1}^{1}$ thin set, i.e., there is a $\Pi_{1}^{1}$ thin subset $C_{1}$ of $X$ such that if $A$ is a $\Pi_{1}^{1}$ thin subset of $X$ then $A \subseteq C_{1}$. A theorem of Kechris (Theorem 1A-2 [7]) gives a sufficient condition for the existence of such largest thin sets with respect to a given hereditary family of subsets of $X$ (in our case, the family of closed smooth sets). The two conditions are: The family has to be $\Pi_{1}^{1}$ on 
the codes of $\Sigma_{1}^{1}$ set and it has to be $\Pi_{1}^{1}$-additive (see [7] for the definition). Since sparse sets have measure zero with respect to the collection of non-atomic, ergodic measures then they are $\Pi_{1}^{1}$-additive ([7]) and from Theorem 2.7 (ii) we get that the other condition is also satisfied. Hence we have the following.

Theorem 3.2. Let $E$ be a $\Delta_{1}^{1}$ non-smooth equivalence relation. There exists a largest $\Pi_{1}^{1}$ sparse set.

\section{The Case of a countable Borel equivalence relation}

In this section we will look at the particular case of a countable Borel equivalence relation, i.e., one for which every equivalence class is countable. Typical examples are equivalence relations generated by a Borel homeomorphism (i.e., hyperfinite equivalence relations [3]), and more generally by the action of a countable group of Borel homeomorphisms. The $\sigma$-ideal of smooth sets with respect to a hyperfinite equivalence relation is the $\sigma$-ideal generated by the wandering sets ([16]).

For a countable Borel equivalence relation $E$ a Borel set $A$ is smooth iff there is a Borel transversal for $A$ ([2]), i.e., there is a Borel transversal $T \subseteq[A]_{E}$ such that $[A]_{E}=[T]_{E}$.

A theorem of Feldman-Moore ([5]) says that for every countable Borel equivalence relation $E$ on a Polish $X$ there is a countable group $G$ of Borel homeomorphisms of $X$ such that $E=E_{G}$, where

$$
x E_{G} y \text { if and only if } g(x)=y \text {, for some } g \in G \text {. }
$$

It is a classical fact that for every Borel subset $B$ of $X$ there is a Polish topology $\tau$, extending the given topology of $X$, for which $B$ is $\tau$-clopen. Moreover, $\tau$ admits a basis consisting of Borel sets with respect to the original topology of $X$. Thus the Borel structures of $X$ and $(X, \tau)$ are the same. As a corollary we get that for every countable Borel equivalence relation $E$ there is a Polish topology $\tau$ and a countable group $G$ of $\tau$-homeomorphisms of $X$ such that $E=E_{G}, \tau$ extends the original topology of $X$ and the Borel structure of $X$ remains the same. These results have an effective version and the FeldmanMoore result quoted above has an effective proof; that is to say: If $E$ is a $\Delta_{1}^{1}$ countable equivalence relation, then there is a countable group $G$ of $\Delta_{1}^{1}$ homeomorphisms of $X$ such that $E=E_{G}$. Moreover, there is a $\Delta_{1}^{1}$ relation $R(x, y, n)$ on $X \times X \times \omega$ such that for all $n, R_{n}$ is the graph of some $g \in G$. And vice versa, for all $g \in G$ there is $n$ such that graph $(g)=R_{n}$. By an abuse of the language we will say that the relation $R(x, y, g) \Leftrightarrow g(x)=y$ is $\Delta_{1}^{1}$. Notice that in this case if $Q(x)$ is a $\Delta_{1}^{1}$ relation, then $\exists g \in G Q(g(x)), \forall g \in$ $G Q(g(x))$ are also $\Delta_{1}^{1}$. In other words $\exists y \in[x]_{E} Q(y)$ and $\forall y \in[x]_{E} Q(y)$ are $\Delta_{1}^{1}$.

If $R(x, y, g)$ is a $\Delta_{1}^{1}$ representation (as above) of the action of $G$ over $X$, then there is a Polish topology $\tau$ extending that on $X$ such that every $g \in G$ is a $\tau$-homeomorphism and $\tau$ admits a basis of $\Delta_{1}^{1}$ sets effectively enumerated. The classical proofs of this fact can be found in [5] and [16], and for the effective counterpart see [13] and [17]. As a corollary of this result we have 
Lemma 4.1. The collection of $\Delta_{1}^{1}$ sets forms a basis for a Polish topology $\tau$ such that every $\Delta_{1}^{1}$ set is $\tau$-clopen.

Lemma 4.2. Let $E$ be a $\Delta_{1}^{1}$ countable equivalence relation on $X, B \subseteq X$ a $\Delta_{1}^{1}$ set and $G$ a countable group of $\Delta_{1}^{1}$ homeomorphisms of $X$ such that $E=E_{G}$ with " $g(x)=y$ " a $\Delta_{1}^{1}$ relation (as it was explained above). There is a Polish topology $\tau$ extending that on $X$ such that every $g \in G$ is a $\tau$-homeomorphism and $[B]_{E}$ is $\tau$-clopen. Moreover, $\tau$ admits a basis of $\Delta_{1}^{1}$ sets effectively enumerated.

The following definitions will play a crucial role in the sequel.

Definition 4.3. Let $\tau$ be a Polish topology on $X$ and put

$$
P(\tau)=\left\{x \in X:[x]_{E} \text { has an isolated point with respect to } \tau\right\} .
$$

If $E$ is generated by a single homeomorphism of $(X, \tau)$, then points not in $P(\tau)$ are the recurrent points of [16]. Recall that for each countable collection $\mathscr{A}=\left(A_{n}\right)$ of $E$-invariant sets we have defined an equivalence relation $x E_{\mathscr{A}} y$ by

$$
x E_{\mathscr{A}} y \text { if and only if } \forall n\left(x \in A_{n} \longleftrightarrow y \in A_{n}\right)
$$

and we denote the $E_{\mathscr{A}}$-equivalence classes by $[x]_{\mathscr{A}}$.

Definition 4.4. For each countable collection $\mathscr{A}=\left(A_{n}\right)$ of $E$-invariant sets put

$$
D_{\mathscr{A}}=\left\{x \in X:[x]_{E}=[x]_{\mathscr{A}}\right\}
$$

i.e., $x \in D_{\mathscr{A}}$ if and only if $\forall y\left(x E y \longleftrightarrow x E_{\mathscr{A}} y\right)$.

Notice that a set $B$ is strongly separated by $\mathscr{A}$ if and only if $B \subseteq D_{\mathscr{A}}$. The following result will be useful in the sequel.

Lemma 4.5. Let $E$ be a countable equivalence relation on $X, \tau$ a Polish topology on $X$ with basis $\left\{W_{n}: n \in \mathbf{N}\right\}$ such that the E-saturation of every $\tau$-open set is $\tau$-open. Put $B_{n}=\left[W_{n}\right]_{E}$ and $\mathscr{B}=\left(B_{n}\right)$. Then $P(\tau)=D_{\mathscr{B}}$.

Proof. First we prove that if $y \notin D_{\mathscr{B}}$, then $y \notin P(\tau)$. It suffices to show that if $x \notin D_{\mathscr{G}}$ and $x \in W_{n}$, then $\left|W_{n} \cap[x]_{E}\right|>1$. This is because if $y \notin D_{\mathscr{B}}$ and $W_{n} \cap[y]_{E} \neq \varnothing$, say $x \in W_{n} \cap[y]_{E}$, then as $D_{\mathscr{B}}$ is invariant $x \notin D_{\mathscr{B}}$, and so $\left|W_{n} \cap[y]_{E}\right|=\left|W_{n} \cap[x]_{E}\right|>1$.

So, suppose $x \notin D_{\mathscr{B}}$ and let $y$ be such that $x E_{\mathscr{B}} y$ but $x \not B y$. Let $n$ be such that $x \in W_{n}$. So, in particular $W_{n} \neq\{x\}$, otherwise $x \in D_{\mathscr{B}}$ (notice that $(X, \tau)$ can have isolated points). As $y \in\left[W_{n}\right]_{E}$, there is $w \in W_{n}$ with $y E w$. Clearly $x \not B w$ and $x E_{\mathscr{B}} w$. Put $V=\left[W_{n}\right]_{E}-\{x\}$, then $V$ is $\tau$-open and $V \cap W_{n} \neq \varnothing$. Thus there is $m$ such that $w \in W_{m} \subseteq V \cap W_{n}$, but as $x E_{\mathscr{B}} w$ then $x \in\left[W_{m}\right]_{E}$. Therefore for some $z \in W_{m} \quad z E x$. Clearly $x \neq z$, hence $\left|W_{n} \cap[x]_{E}\right|>1$, i.e., $x \notin P(\tau)$.

Now we show that if $x \in D_{\mathscr{B}}$ then $x \in P(\tau)$. Let $x \in D_{\mathscr{B}}$, then $[x]_{E}=$ $[x]_{\mathscr{B}}$ and hence $[x]_{E}=\left\{y: \forall n\left(x \in B_{n} \leftrightarrow y \in B_{n}\right)\right\}$. As each $B_{n}$ is $\tau$-open, $[x]_{E}$ is a $\tau-G_{\delta}$ set. Since $[x]_{E}$ is countable, by the Baire category theorem we conclude that $[x]_{E}$ has a $\tau$-isolated point, i.e., $x \in P(\tau)$.

Notice that $P(\tau) \subseteq D_{\mathscr{B}}$ is always true, without assuming that $E$ is countable. 
Lemma 4.6. Let $\tau$ be a Polish topology on $X$ with a basis consisting of Borel sets with respect to the original topology on $X$. Let $G$ be a countable group of $\tau$-homeomorphisms of $X$ and $E=E_{G}$. Then $a \tau$ - $G_{\delta}$ E-invariant set $H$ is $E$-smooth if and only if $H \subseteq P(\tau)$.

Proof. Let $\mathscr{B}$ be as in Lemma 4.5. Then $P(\tau) \subseteq D_{\mathscr{B}}$. As each element of the basis of $\tau$ is Borel, we get that $P(\tau)$ is strongly Borel separated.

On the other hand, suppose $H$ is $E$-smooth, by a result of Effros [4] we get that for every $x \in H,[x]_{E}$ is $\tau$-locally closed in $H$. But as $H$ is $\tau-G_{\delta}$ and $[x]_{E}$ is countable, then $[x]_{E}$ has a $\tau$-isolated point, i.e., $x \in P(\tau)$.

We get the following characterization of Borel smooth sets.

Theorem 4.7. Let $E$ be a Borel countable equivalence relation on $X$ and $B$ a Borel subset of $X$. Let $\tau_{B}$ be the Polish topology for $[B]_{E}$ given by Lemma 4.2. Then $B$ is smooth if and only if $B \subseteq P\left(\tau_{B}\right)$.

Proof. Since $[B]_{E}$ is $\tau_{B}$-clopen, by Lemma $4.6[B]_{E}$ is smooth if and only if $[B]_{E} \subseteq P\left(\tau_{B}\right)$. And by Theorem $2.3 B$ is smooth if and only if $[B]_{E}$ is smooth. Finally observe that $P(\tau)$ is an invariant set; thus $B \subseteq P\left(\tau_{B}\right)$ if and only if $[B]_{E} \subseteq P\left(\tau_{B}\right)$.

Remark. (1) Theorem 4.7 can be seen as a Borel analog of Theorem 2.7 (iii). That is to say, for Borel smooth sets $P(\tau)$ plays the same role as $D$ does for $\Sigma_{1}^{1}$ smooth sets. We will show below that $D=P(\tau)$ for some topology.

(2) On the other hand this is a generalization of a result of Weiss [16] which says that the equivalence relation induced by an aperiodic homeomorphism is not smooth if and only if there is a recurrent point.

(3) From this result one can easily get that every Borel $E$-smooth set $B$ admits a Borel transversal (this is a well-known result of Burgess which holds for actions of Polish groups [2]). In fact, let $\left\{W_{n}\right\}$ be a basis for the topology $\tau_{B}$ (as in Theorem 4.7) and define $R(n, x)$ if and only if $n$ is the least $m$ (if it exists) such that $\left|W_{m} \cap[x]_{E}\right|=1$. It is not difficult to show that $R$ is Borel and clearly $P(\tau)=\exists^{\omega} R$. Define $T$ by $x \in T$ iff $\exists m R(m, x) \& x \in W_{m}$. It is easy to check that $T$ is a transversal for $P\left(\tau_{B}\right)$ and hence $T \cap[B]_{E}$ is a transversal for $[B]_{E}$.

Our next theorem answers a question raised in $\S 2$.

Theorem 4.8. Let $E$ be a countable $\Delta_{1}^{1}$ equivalence relation on $X$. Let $D$ be the set defined on Theorem 2.7(iii) and $\rho$ be the Polish topology generated by the $\Delta_{1}^{1}$ sets given by Lemma 4.1. Then

(i) $D=P(\rho)$.

(ii) $D=\bigcup\left\{A: A\right.$ is a $\Delta_{1}^{1}$ smooth set $\}$.

Proof. Let us show first that (i) implies (ii). Let $x \in D$. We want to show that there is a $\Delta_{1}^{1}$ smooth set $A$ with $x \in A$. Since $x \in P(\rho)$ then $[x]_{E}$ has a $\rho$-isolated point. Let $B$ be a $\Delta_{1}^{1}$ set such that $\left|B \cap[x]_{E}\right|=1$. Put $A=\left\{y:\left|B \cap[y]_{E}\right|=1\right\}$. Since $E$ is the action of a countable group and the action is $\Delta_{1}^{1}$ (as in the hypothesis of Lemma 4.2) then $A$ is $\Delta_{1}^{1}$. Clearly $A \subseteq P(\rho)=D$, so $A$ is smooth and $x \in A$.

Let $\mathscr{A}=\left(A_{n}\right)$ be the collection of $\Delta_{1}^{1}$ invariant sets. It follows from the proof of Lemma 2.5 that $D=D_{\mathscr{A}}$. For every $\Delta_{1}^{1}$ set $A,[A]_{E}$ is $\Delta_{1}^{1}$. Hence from Lemma 4.2 and Lemma 4.5 we get that $D=P(\rho)$. 
As we have observed before, the previous theorem implies that strong Borel separation and smoothness are equivalent.

Theorem 4.9. Let $E$ be a $\Delta_{1}^{1}$ countable equivalence relation on $X$ and $C$ be an arbitrary subset of $X$. Then $C$ is smooth if and only if $C$ is strongly Borel separated.

Proof. (i) $\Rightarrow$ (ii) is a consequence of Theorem 2.3 , as $\Delta_{1}^{1}$ smooth sets are clearly strongly $\Delta_{1}^{1}$-separated.

(ii) $\Rightarrow$ (i). Let $C$ be a strongly $\Delta_{1}^{1}$-separated set. Since $D$ is the largest $\Delta_{1}^{1}$ separated set (see the remark after the proof of Theorem 2.7) then $C \subseteq D$ and from Theorem 4.8 we have that $D$ is Borel.

Remark. From Theorem 3.1 we get that this result is not valid if we replace strong separation by separation.

To finish this section we will compare smoothness and category. It is easy to define a Borel equivalence relation for which there is a smooth dense $G_{\delta}$ set, and in consequence smoothness does not necessarily imply meagerness. One example is the following: Let $F$ be a non- $E_{0}$-smooth $F_{\sigma}$ set of first category (for instance, the saturation of any non-smooth closed meager set) and define an equivalence relation $E$ as follows:

$$
x E y \text { if and only if } x=y \text { or }\left(x, y \in F \& x E_{0} y\right) \text {. }
$$

Then $E$ is a countable non smooth equivalence relation. Let $H=2^{\omega}-F$. Then $H$ is $G_{\delta}$ dense $E$-transversal. However, for some equivalence relations every smooth set is of first category, as we will show next.

Let $G$ be a collection of homeomorphisms of $X$. We will say that $G$ satisfies the condition $(*)$ if the following holds: For every open set $O$ there exists $g \in G$ and $x \in O$ such that $g[O]=O$ and $g(x) \neq x$.

For instance $E_{0}$ is generated by the following collection of homeomorphisms of $2^{\omega}$ : For each $s, t \in 2^{n}, n \in \mathbf{N}$ let $f_{s, t}$ defined by:

$$
f_{s, t}(\alpha)= \begin{cases}t^{\wedge} \gamma & \text { if } \alpha=s^{\wedge} \gamma \\ s^{\wedge} \gamma & \text { if } \alpha=t^{\wedge} \gamma, \\ \alpha & \text { otherwise. }\end{cases}
$$

Where $t^{\wedge} \gamma$ denotes the concatenation of $t$ followed by $\gamma$. It is clear that each $f_{s, t}$ is an homeomorphism. This collection generates $E_{0}$ and satisfies (*).

Lemma 4.10. Let $E$ be an equivalence relation on $X$ generated by a collection $G$ of homeomorphisms of $X$ which satisfies condition (*). If $O$ is an open set and $H \subset O$ is a dense (in $O) G_{\delta}$ set then $H$ is not a transversal.

Proof. By (*) there is $g \in G$ such that $g[O]=O$. Let $H_{1}=g^{-1}[H]$. Then $H_{1}$ is a dense $G_{\delta}$ subset of $O$ and so is $H_{2}=H_{1} \cap H$. By $(*)$ there is $z \in H_{2}$ with $g(z) \neq z$, i.e. $H_{2}$ is not a transversal.

If $E$ is countable and Borel, every smooth set admits a Borel transversal (see part (3) of the remark after Theorem 4.7) and therefore we get the following:

Theorem 4.11. Let $E$ be an equivalence relation generated by a collection $G$ of homeomorphisms of $X$ which satisfies (*). Then

(i) Every E-transversal with the property of Baire is of first category. 
(ii) If in addition $G$ is countable and $E$ is Borel, then every $E$-smooth set is of first category.

Corollary 4.12. Every $E_{0}$-smooth set is of first category.

\section{THE $\sigma$-IDEAL OF CLOSED SMOOTH SETS}

As we have already pointed out, Theorem 2.3 implies that the notion of smoothness for $\Sigma_{1}^{1}$ sets is concentrated on closed sets, i.e., a $\Sigma_{1}^{1}$ set $A$ is smooth if and only if every closed subset of $A$ is smooth. In this section we will present some properties of the collection of closed smooth sets.

The collection of closed subsets of $X$, which is denoted by $\mathscr{K}(X)$, equipped with the Hausdorff distance is a Polish space. All the notions such as open sets, Borel sets, analytic sets, etc., in $\mathscr{K}(X)$ will refer to the Hausdorff metric (for more details about the topology on $\mathscr{K}(X)$ see [14] and the references given there).

Let $E$ be a Borel equivalence relation on a compact Polish space $X$ and let

$$
I(E)=\{K \in \mathscr{K}(X): K \text { is smooth with respect to } E\} .
$$

It is clear that $I(E)$ is a $\sigma$-ideal of compact sets (i.e., the following two properties hold: (1) If $K_{n} \in I(E)$ for all $n \in \omega$ and $K=\bigcup_{n} K_{n}$ is closed then $K \in I(E)$. (2) $I$ is hereditary, i.e., if $K \in I(E)$ and $F \subseteq K$ is closed then $F \in I(E)$ ). There has been much interest in the study of $\sigma$-ideals of compact sets since it was discovered its connection with harmonic analysis ([12]). Many descriptive set theoretic properties of $\sigma$-ideals of compact sets have been investigated and shown to be quite interesting (see [14], [12], [9], [8], [18]). We are interested in studying the complexity of $I(E)$ as well as some structural properties such as calibration, the covering property and existence of Borel basis. One of the results of this section is that $E$ is smooth if and only if $I(E)$ is Borel. We will also look at the particular case of $I\left(E_{0}\right)$.

First we will recall some basic facts about $\sigma$-ideals. A $\Pi_{1}^{1} \sigma$-ideal $I$ satisfies the so-called dichotomy theorem ([14]), namely either $I$ is a true $\Pi_{1}^{1}$ subset of $\mathscr{K}(X)$ or a $G_{\delta}$ subset. Even more, every $\Sigma_{1}^{1} \sigma$-ideal is in fact $G_{\delta}$ ([14]). A $\sigma$-ideal $I$ is strongly calibrated if for every closed set $F \subseteq X$ with $F \notin I$ and every $\Pi_{2}^{0}$ set $H \subseteq X \times 2^{\omega}$ such that $\operatorname{proj}(H)=F$, there is a closed set $K \subseteq H$ such that $\operatorname{proj}(K) \notin I$. We say that $B \subset I$ is a basis for $I$ if $B$ is hereditary and $I=B_{\sigma}$, i.e., every $K \in I$ is a countable union of sets in $B$. We say that $I$ has Borel basis if there is a Borel subset of $\mathscr{K}(X)$ which is a basis for $I$. I is called locally non-Borel if for every closed set $F \notin I, I \cap \mathscr{K}(F)$ is not Borel. We say that $I$ is thin if every collection of disjoint closed sets not in $I$ is at most countable. These notions were introduced in [14].

Theorem 5.1. Let $E$ be a non-smooth $\Delta_{1}^{1}$ equivalence relation on a compact Polish space $X$. Then $I(E)$ is a strongly calibrated, locally non-Borel, non-thin $\Pi_{1}^{1} \sigma$-ideal.

We will need the following lemmas.

Lemma 5.2. Let $f: 2^{\omega} \rightarrow X$ be a continuous embedding from $E_{0}$ into $E$. For every closed set $K \subseteq 2^{\omega}$ 
$K \in I\left(E_{0}\right)$ if and only if $f[K] \in I(E)$.

Proof. Let $K \notin I\left(E_{0}\right)$ and put $E_{1}=E_{0}\left\lceil K\right.$. By Theorem 2.3, $E_{0} \sqsubseteq E_{1}$ via a continuous embedding. But clearly $E_{1} \sqsubseteq E\lceil f[K]$ and $\sqsubseteq$ is transitive. Hence $E_{0} \sqsubseteq E\lceil f[K]$, i.e., $f[K] \notin I(E)$.

Conversely, suppose $K \in I\left(E_{0}\right)$ and let $\mathscr{A}=\left(A_{n}\right)$ be a separating family of $\Sigma_{1}^{1}$ sets for $E_{0}\left[K\right.$. Put $B_{n}=f\left[A_{n}\right]$ and $\mathscr{B}=\left(B_{n}\right)$. We claim that $\mathscr{B}$ is a separating family for $E[f[K]$. In fact, as $f$ is $1-1$ we have that $\forall x, y\left(f(x) E_{\mathscr{B}} f(y) \leftrightarrow x E_{\mathscr{A}} y\right)$. Hence $\forall z, w \in f[K]\left(z E_{\mathscr{B}} w \leftrightarrow z E w\right)$.

Lemma 5.3. For every $x \in 2^{\omega}$ there is a continuous map $f: 2^{\omega} \rightarrow \mathscr{H}\left(2^{\omega}\right)$ such that

(i) if $\gamma$ is eventually zero, then $f(\gamma)$ is a finite subset of $[x]_{E_{0}}$.

(ii) if $\gamma$ is not eventually zero, then $f(\gamma)$ is a non-smooth closed set (with respect to $\left.E_{0}\right)$.

In other words, there is a continuous reduction of $\left\{\alpha \in 2^{\omega}: \alpha\right.$ is eventually zero $\}$ into the collection of finite subsets of $[x]_{E_{0}}$ and $\sim I\left(E_{0}\right)$. In particular $I\left(E_{0}\right)$ is not $G_{\delta}$.

Proof. Consider the following function:

$$
f(\gamma)=\left\{\alpha \in 2^{\omega}: \forall n(\gamma(n)=0 \rightarrow \alpha(n)=x(n))\right\} .
$$

Clearly if $\gamma$ is eventually zero, then (i) holds. On the other hand if $\gamma$ has infinite many 1's, then $f(\alpha)$ is a perfect set. Let $g: 2^{\omega} \rightarrow 2^{\omega}$ be the canonical bijection of $2^{\omega}$ onto $f(\gamma)$. It is not difficult to see that $g$ is actually an embedding from $E_{0}$ into $E_{0}\left\lceil f(\gamma)\right.$, i.e., for all $\alpha, \beta \in 2^{\omega}, \alpha E_{0} \beta$ if and only if $g(\alpha) E_{0} g(\beta)$ (just observe that if $T$ is the tree of $f(\gamma)$ and some sequence in $T$ of length $n$ splits, then every sequence in $T$ of length $n$ splits).

Finally, to see that $f$ is continuous, let for each $s \in 2^{<\omega}$

$$
A_{s}=\left\{\alpha \in 2^{\omega}: \forall n<\operatorname{lh}(s)(s(n)=0 \Rightarrow \alpha(n)=x(n))\right\},
$$

each $A_{s}$ is closed and if $t \prec s$, then $A_{s} \subseteq A_{t}$. We have that $f(\gamma)=\bigcap_{n} A_{\gamma\lceil n}$ and also that for every $s \in 2^{<\omega}$

$$
f(\gamma) \cap N_{s} \neq \varnothing \quad \text { if and only if } \forall n<\ln (s)(s(n)=0 \Rightarrow \gamma(n)=x(n))
$$

which easily implies that $f$ is continuous. Since $\left\{\alpha \in 2^{\omega}: \alpha\right.$ is eventually zero $\}$ is countable and dense then by the Baire category theorem $I\left(E_{0}\right)$ is not $G_{\delta}$.

Proof of Theorem 5.1. It is clear that $I(E)$ is a $\sigma$-ideal and since the smooth sets are the common null sets of all $E$-ergodic, non-atomic measures on $X$, by a standard capacitability argument (see for instance, [18], page 126) we get that $I(E)$ is strongly calibrated. A similar argument as in the proof of (ii) in Theorem 2.7 shows that $I(E)$ is $\Pi_{1}^{1}$.

First, notice that from the dichotomy theorem for $\sigma$-ideals ([14]) and 5.3 we get that $I\left(E_{0}\right)$ is not Borel. To see that $I(E)$ is locally not Borel let $K \in \mathscr{K}(X)$, we then have that

$$
I(E) \cap \mathscr{K}(K)=\{F \in \mathscr{K}(K): F \text { is } E \text {-smooth }\}=I(E\lceil K) .
$$

From Lemma 5.2 we get that $I\left(E_{0}\right)$ is not Borel if and only if $I(E\lceil K)$ is not Borel.

Finally, to show that $I(E)$ is not thin, clearly it is enough to show that for $E_{0}$. Let $I$ be a $\sigma$-ideal, a result of [18] (Theorem 2.5) says that if every 
set in $I$ is meager and $I$ is thin then there is a dense $G_{\delta}$ set $G$ such that $\mathscr{K}(G) \subseteq I$. From Corollary 4.12 every $E_{0}$-smooth set is meager, so $I\left(E_{0}\right)$ cannot be thin.

As a corollary of Lemma 5.3 we get the following

Corollary 5.4. Let $E$ be a non-smooth Borel equivalence relation on $X$, then

(i) If $J \subseteq I\left(E_{0}\right)$ is a dense $\sigma$-ideal, then $J$ is not $\Sigma_{1}^{1}$.

(ii) If $J \subseteq I(E)$ is a $\sigma$-ideal such that for every $x \in X \quad\{x\} \in J$, then $J$ is not $\Sigma_{1}^{1}$.

Proof. (ii) follows from (i), because if $f: 2^{\omega} \rightarrow X$ is an embedding witnessing that $E$ is not smooth and $J \subseteq I(E)$ is a $\sigma$-ideal containing all singletons, then $J^{*}=f^{-1}[J]$ is a dense $\sigma$-ideal and it is contained in $I\left(E_{0}\right)$ (by Lemma 5.2).

(i) Let $J$ be as in the hypothesis of (i). Every $\Sigma_{1}^{1} \sigma$-ideal is actually $G_{\delta}$ ([14]). Hence if suffices to show that $J$ is not $G_{\delta}$. Suppose toward a contradiction that $J \subseteq I\left(E_{0}\right)$ is a $G_{\delta}$ dense $\sigma$-ideal. Let $H=\left\{x \in 2^{\omega}:\{x\} \in J\right\}$, $H$ is a $G_{\delta}$ dense set. Let $G$ be a countable collection of homeomorphisms of $2^{\omega}$ generating $E_{0}$. Put $H^{*}=\bigcap_{g \in G} g[H], H^{*}$ is an invariant dense $G_{\delta}$ subset of $H$. Let $x \in H^{*}$, for every $y E_{0} x$, we have $\{y\} \in J$. But from Lemma 5.3, such $J$ cannot be a $G_{\delta}$ set, a contradiction.

From Theorem 5.1 we get the following characterization of a smooth Borel equivalence relation.

Corollary 5.5. Let $E$ be a Borel equivalence relation on $X$. Then $E$ is smooth if and only if $I(E)$ is Borel.

Remark. (1) Corollary 5.4 (ii) above is the best possible in the following sense: We have seen in $\S 3$ that there is a non-smooth Borel equivalence relation $E$ and a dense $G_{\delta}$ set $H$ which is $E$-smooth. Clearly $\mathscr{K}(H)$ is a Borel dense subideal of $I(E)$.

(2) Kechris ([9]) has proved that the $\sigma$-ideal of closed sets of extended uniqueness also satisfies this hereditary property but even in a stronger form, i.e., for every perfect set $M$ of restricted multiplicity the $\sigma$-ideal $U_{0} \cap \mathscr{K}(M)$ has no dense $\Sigma_{1}^{1}$ subideals. We do not know if this holds for $I\left(E_{0}\right)$.

Another structural property that has been studied in the context of $\sigma$-ideals of compact sets is the so called covering property (see [8], [18]). This is a quite strong property and there are few known $\sigma$-ideals that have it. Theorem 3.1 suggests that $I(E)$ does not have the covering property. We will address this question in a forthcoming paper.

\section{REFERENCES}

1. H. Becker, The restriction of a Borel equivalence relation to a sparse set, preprint, 1992.

2. J. Burgess, A selection theorem for group actions, Pacific J. Math. 80 (1979), 333-336.

3. R. Dougherty, S. Jackson and A. S. Kechris, The structure of hyperfinite Borel equivalence relations, Trans. Amer. Math. Soc. 341 (1994), 193-225.

4. E. Effros, Polish transformation groups and classification problems, General Topology and Modern Analysis, (M. M. Rao and L. F. Mc Auley, eds.), Academic Press, 1980, pp. $217-$ 227.

5. J. Feldman and C. C. Moore, Ergodic equivalence relations, cohomology and von Neumann algebras, I, Trans. Amer. Math. Soc. 234 (1977), 289-324. 
6. L. A. Harrington, A. S. Kechris and A. Louveau, A Glimm-Effros dichotomy for Borel equivalence relations, J. Amer. Math. Soc. 3 (1990), 903-928.

7. A. S. Kechris, The theory of countable analytical sets, Trans. Amer. Math. Soc. 202 (1975), 259-297.

8. The descriptive set theory of $\sigma$-ideals of compact sets, Logic Colloquium'88, ( $\mathbf{R}$. Ferro, C. Bonotto, S. Valentini and A. Zanardo, eds.), North-Holland, 1989, pp. 117-138.

9. $\ldots$, Hereditary properties of the class of closed uniqueness sets, Israel J. Math. 73 (1991), 189-198.

10. 337-384.

11. $\ldots$, Amenable versus hyperfinite Borel equivalence relations, J. Symbolic Logic 58 (1993), 894-907.

12. A. S. Kechris and A. Louveau, Descriptive set theory and the structure of sets of uniqueness, LMS Lecture Notes, 128, Cambridge Univ. Press, 1987.

13. A.S. Kechris, Lectures on Borel equivalence relation, unpublished.

14. A. S. Kechris, A. Louveau and W. H. Woodin, The structure of $\sigma$-ideals of compact sets, Trans. Amer. Math. Soc. 301 (1987), 263-288.

15. Y. N. Moschovakis, Descriptive set theory, North-Holland, Amsterdam, 1980.

16. B. Weiss, Measurable dynamics, Contemp. Math. 26 (1984), 395-421.

17. C. Uzcátegui, Ph.D. Thesis, Caltech, 1990.

18. The covering property for $\sigma$-ideals of compact sets, Fund. Math. 140 (1992), 119-146.

Universidad de los Andes, Facultad de Ciencias, Departamento de Matemáticas, MERIDA 5101, Venezuela

E-mail address: uzca@ciens.ula.ve 